\title{
Systematic parameter study of a nonlinear electromagnetic energy harvester with matched magnetic orientation: numerical simulation and experimental investigation
}

\author{
Wei Deng and Ya Wang* \\ Department of Mechanical Engineering, Stony Brook University, Stony Brook 11790, USA
}

\begin{abstract}
This paper reports the systematic parameter study of a tristable nonlinear electromagnetic energy harvester for ambient low-frequency vibration. Numerical simulations and experimental investigations are performed on the harvester which consists of a cantilever beam, a tip coil, two tip magnets and two external side magnets. The external side magnets are deployed symmetrically along a concave surface parallel to the trajectory of the cantilever tip with a controllable distance so that the magnetic orientation of the tip magnets are matched with that of the side magnets. Therefore, instead of the ternary position parameters $(d, h, \alpha)$, a binary parameters pair $\left(d_{0}, d\right)$ is used to characterize the position of the side magnets and the performance of the energy harvester. The magnetic force and magnetic field on the cantilever tip therefore depend on the relative distance in the tip displacement direction between the tip magnets and side magnets, but is independent of the position of the side magnets on the concave surface. The magnetic force (field)-distance relationship is measured experimentally and curve fitted to obtain explicit expressions, in order to characterize the magnetic force (field) when the side magnets are placed at varied positions along the concave surface. Numerical simulation is, then, performed to predict the electromagnetic voltage output and the bandwidth of the energy harvester. The simulation results coincided with the measured data. Significant broadband response is obtained experimentally and the maximum RMS power output is $40.2 \mathrm{~mW}$ at $0.45 \mathrm{~g}$ of excitation. The proposed structure showcasing the matched magnetic orientation is characterized by the binary parameters pair $\left(d_{0}, d\right)$ and the systematic parametric approach could contribute to the design and study of nonlinear broadband energy harvesters.
\end{abstract}

Keywords: tristable nonlinear electromagnetic energy harvester, matched magnetic orientation, systematic parameter analysis

\section{Introduction}

Low-frequency vibrational energy is widely available in an ambient environment and could be harvested to power remote microsystems. The most prolific structure consists of a cantilever metallic beam with harvesting units, such as piezoelectric or electromagnetic transducers. With high voltage but small current output, piezoelectric transducers have been theoretically and experimentally studied over the past couple of decades [16]. On the contrary, one may prefer electromagnetic transducers when a low voltage but large current output is required in the application [7-9]. In addition to the conversion mechanism, bandwidth limitation is a more pressing issue since the ambient vibrational energy is either distributed over a wide spectrum of frequencies or the dominant frequency slowly drifts away with respect to time. The application of a linear energy harvester (LEH) is thus limited, as its efficiency will drop substantially in the event that the excitation frequency slightly mismatches its fundamental resonant frequency. One promising solution is to apply external design elements that introduces a nonlinear magnetic force to the LEH, modifying the restoring force and the potential energy profile, in order to obtain a broadband response of a nonlinear energy harvester (NEH). For example, the nonlinear stochastic oscillation of a noise activated energy harvester could enhance the power output performance by about $200 \%$ compared to its standard LEH counterpart in terms of the power output [10, 11]. The performance superiority of a nonlinear piezomagnetoelastic energy harvester (consisting of a ferroic beam

\footnotetext{
* Corresponding author: Ya Wang, Assistant Professor, Department of Mechanical Engineering, State University of New York, Stony Brook, NY, USA, Phone: 631-632-8322, Email: ya.s.wang@stonybrook.edu
} 
with external magnets) as a high-energy large orbit attractor has been experimentally and theoretically proven [12]. A vibrational power flow analysis approach has been adopted to assess the long-time behavior of a nonlinear system exhibiting either periodic or chaotic motions [13]. A thorough investigation of a bistable piezoelectric inertial generator has also been carried out to study the favorable nature of strange nonlinear chaotic motions [14]. It has been revealed that it is possible to define the minimum excitation variance that enhances the harvested energy for a given bistable piezoelectric generator [15].

Another NEH configuration proposed by Stanton et al. consists of two magnets attached at the end of the cantilever beam and two external side magnets deployed symmetrically [16]. With properly designed position parameters (transverse displacement $d$, and longitudinal displacement $h$ ) of the side magnets, the proposed NEH is capable of harvesting energy more efficiently than a LEH from excitations with a slow-frequency drift around its respective dominant resonance in a softening or a hardening configuration. Later on, Zhou et al. introduced another position parameter, angular orientation $\alpha$, which could be adjusted by rotating the side magnets to achieve broadband response at different center frequencies [17]. The NEH could be designed as bistable or tristable by choosing different values for the position parameters $(d, h, \alpha)$, with the tristable NEH demonstrating a better output performance [18]. With the same configuration, a shallower potential well depth is testified to produce a wider bandwidth and a higher harvesting efficiency under low-frequency excitation [19]. Study also shows that compared with a bistable NEH, the tristable NEH could achieve inter-well oscillations at a lower frequency threshold but create a dense and high output power at a low intensity of stochastic excitation [20]. More recently, this configuration with nonlinear time-varying potential is applied to harvest energy from the walking motion of human beings[21,22].

The typical configuration of the aforementioned NEH could produce a monostable, bistable or tristable response depending on the position parameters $(d, h, \alpha)$ of the side magnets. The position parameters are extremely important because they determine the restoring force and feature the nonlinear magneto-elastic interaction. However, work in the (referenced) literature often investigates the position parameters $(d, h, \alpha)$ independently. Moreover, the restoring force is usually measured from multiple experiments and then curve fitted as polynomial functions [17-19, 23] for numerical simulation, which is time-consuming to numerate values for $(d$, $h, \alpha)$ separately and to measure the restoring force in correspondence to each set of values of $(d, h, \alpha)$. Although theoretical formula of the magnetic force could be derived based on the magnetic dipole model [10, 14, 24], this approach is difficult to implement when the configuration of the harvester is complex, due to the fact that when applying three dimensional magnetic interactions, the model does not take into account the shape of the magnet as well.

In this paper, the typical configuration is modified to construct a nonlinear electromagnetic energy harvester (EMEH) where the magnetic orientation of the tip magnets matches that of the side magnets by positioning the side magnets along a concave surface parallel to the motion trajectory of the cantilever tip, and the position parameters $(d, h, \alpha)$ are studied interdependently. The transverse displacement $d$ is selected as the primary parameter, the longitudinal position $h$ and the angular orientation $\alpha$ are then determined simultaneously, therefore, a binary parameters pair $\left(d_{0}, d\right)$, instead of the ternary position parameters $(d, h, \alpha)$, is used to characterize the position of the side magnets and the performance of the EMEH, where $d_{0}$ is the distance between the concave surface and tip trajectory. The magnetic force and the magnetic field on the cantilever tip only depend on the relative distance in the tip displacement direction between the tip magnets and side magnets due to the matched magnetic orientation in the proposed structure. The corresponding restoring force and the magnetic field at varied values of $d$ are calculated based on the measured magnetic force (flux)-distance relation. A systematic parameter analysis and a numerical study are then performed based on the electromagnetic governing equation and the results coincided with the measured data. The optimal power output (RMS) is also measured to verify the energy harvesting capability of the proposed nonlinear EMEH.

\section{Structure of the nonlinear EMEH}

The schematic diagram of the proposed nonlinear EMEH is illustrated in Fig. 1(a). It consists of an aluminum cantilever beam with a coil and two magnets (K\&J Magnetics, $12.7 \mathrm{~mm} \times 6.35 \mathrm{~mm} \times 6.35 \mathrm{~mm}$ ) at the tip. Two 
external magnets (K\&J Magnetics, $25.4 \mathrm{~mm} \times 19.7 \mathrm{~mm} \times 12.7 \mathrm{~mm}$ ) are positioned symmetrically at two sides along an aluminum concave shim. The trajectory of the tip is measured and the aluminum shim is made to have the same curve shape as the tip's trajectory so that the magnetic orientation of the tip magnets is normal to the aluminum shim and it is parallel to the orientation of the side magnets when the tip magnets face the side magnets. A photo representation of the nonlinear EMEH is presented in Fig. 1(b). Since the magnetic force between tip magnets and side magnets is attractive, the structure works as a softening configuration. The mechanical and material properties of the studied EMEH are listed in Table 1.

Table 1 Properties of the proposed EMEH

\begin{tabular}{|ll|}
\hline Size of cantilever & $149.3 \mathrm{~mm} \times 22 \mathrm{~mm} \times 0.81 \mathrm{~mm}$ \\
Young's modulus of cantilever & $68.9 \mathrm{Gpa}$ \\
Density of cantilever beam & $2.7 \mathrm{~g} / \mathrm{cm}^{3}$ \\
Outer size of coil & $22 \mathrm{~mm} \times 22 \mathrm{~mm} \times 9.6 \mathrm{~mm}$ \\
Inner size of coil & $12.5 \mathrm{~mm} \times 12.5 \mathrm{~mm} \times 9.6 \mathrm{~mm}$ \\
Coil turns & 500 \\
Tip mass & $24 \mathrm{~g}$ \\
\hline
\end{tabular}

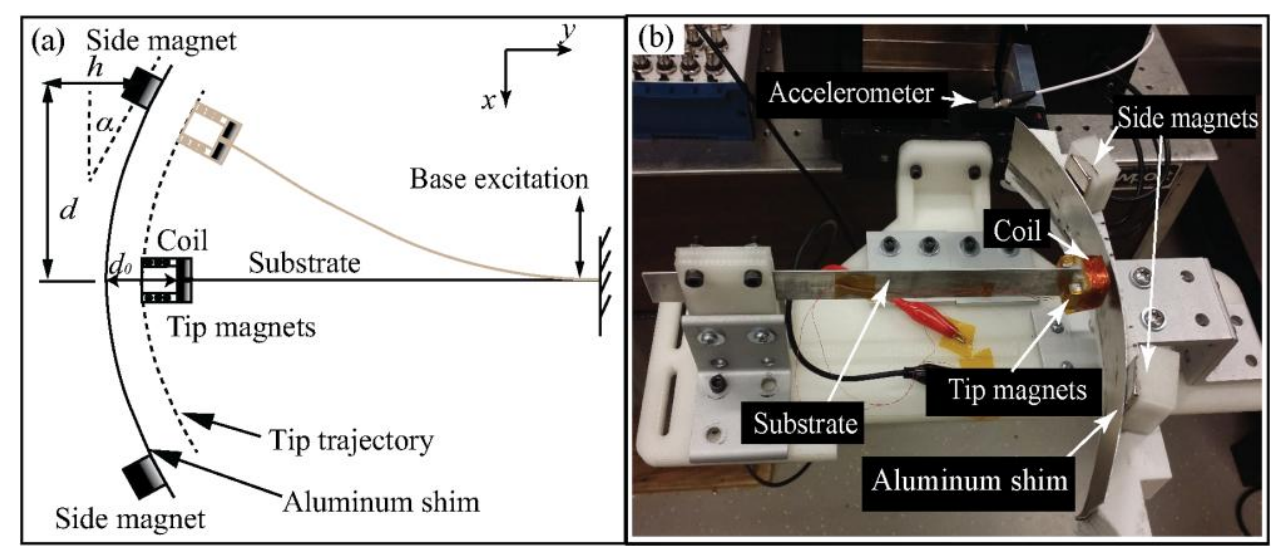

Fig. 1. (a) The schematic diagram illustration and (b) the photo representation of the proposed nonlinear EMEH.

\section{Magnetic force (field) measurement and calculation}

Since the position parameters $(d, h, \alpha)$ are interdependent, only one of them is chosen as the variable. As shown in Fig. 1(a), once the distance $d_{0}$ between the tip magnets and aluminum shim is designated, one parameter is enough to denote the position and angular orientation of the side magnets. In the following study, the transverse displacement $d$ is selected as the variable. As the tip trajectory and concave surface is parallel with respect to each other, what matters for the magnetic force (field) is the relative distance in the $x$-axis between the tip magnets and side magnets, instead of the transverse displacement $d$ or the cantilever tip displacement $x$. The distance-dependent magnetic force is first measured using the diagram shown in Fig. 2(a). Only one of the side magnets is put at the center of the concave surface and the magnetic force in the $x$-axis is measured. The magnetic field at the cantilever tip is also measured normal to the tip trajectory since the electromagnetic voltage is proportional to the derivative of the magnetic flux. The results are shown in Fig. 2(b). In order to calculate the magnetic restoring force and the magnetic field in the nonlinear EMEH when $d$ is varied, explicit expressions are obtained from the measured data. As introduced in Ref [10, 14], the formula of the magnetic force derived from the magnetic dipole model is like a fraction where both the denominator and numerator are functions of the magnets distance. Since the distance is the square root of a second-order polynomial, it is intuitive to use polynomials to approximate the numerator and denominator. In order not to lose generality of the method but also consider the physical origination, the experimental data of the magnetic force are curve fitted using a rational function of $\Delta d$ where the numerator and denominator are polynomials. The magnetic field generated by one magnetic dipole at the location of the other magnetic dipole is proportional to $\left(\vec{\mu} r^{-3}-3(\vec{\mu} \cdot \vec{r}) \vec{r} r^{-5}\right) \cdot \hat{n}$, where $\vec{\mu}$ is the magnetic moment vector and $\vec{r}$ is the vector from one magnetic dipole to the other one, $\hat{n}$ is the 
normalized vector in the axis of the coil. The multiplication of the vectors in the formula implicates that the magnetic field should depend on the angle between the vectors and sinusoidal functions could be used.

Therefore, the measured data of the magnetic field are curve fitted using a sum of sinusoidal functions, which also ensures that the fitting could converge. The fitted expressions are

$$
\left\{\begin{array}{l}
F_{c}=\sum_{i=0}^{4} a_{i}(\Delta d)^{i} / \sum_{j=0}^{3} b_{j}(\Delta d)^{j} \\
B_{c}=\sum_{k=0}^{3} A_{k} \sin \left(B_{k}(\Delta d)+C_{k}\right)
\end{array}\right.
$$

Where $a_{i}, b_{j}, A_{k}, B_{k}$ and $C_{k}$ are the fitted coefficients.

The magnetic restoring force and magnetic field on the cantilever tip of the proposed EMEH shown in Fig. 1 are dependent on $d$ and $x$ because the distance between the tip magnets and side magnets are co-determined by $d$ and $x$, that is, $\Delta d$ is a function of $d$ and $x$. Using Eq. (1) and considering the sign of $x$, the magnetic restoring force and magnetic field at the cantilever tip are:

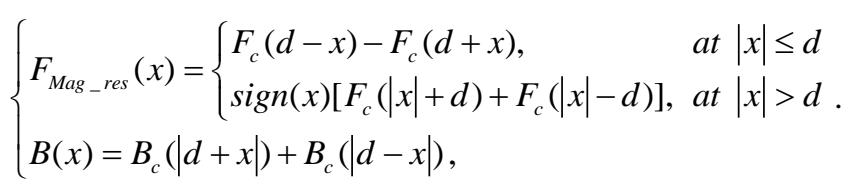

The formulae in Eq. (2) include two terms, $F_{c}(d-x)$ and $F_{c}(d+x), F_{c}(|x|+d)$ and $F_{c}(|x|-d)$, or $B_{c}(|d+x|)$ and $B_{c}(|d-x|)$, that represents the magnetic interaction with each of the side magnets. And Eq. 2 also displays the fact that the two side magnets do not influence each other. The magnetic restoring force and the magnetic field calculated from Eq. (2) are plotted in Fig. 3(a) and (b) to illustrate their dependence on $d$.

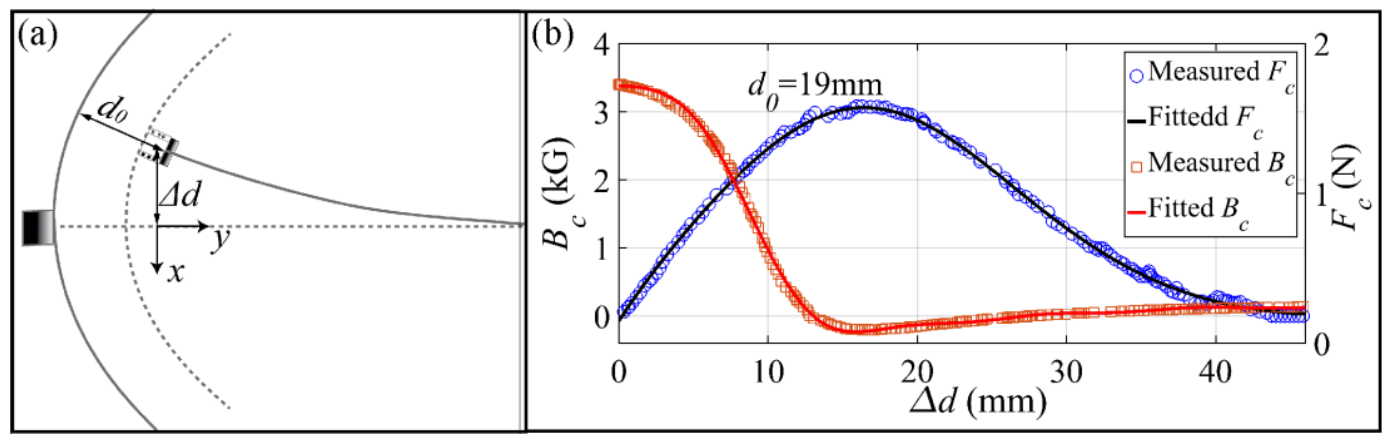

Fig. 2. (a) The schematic diagram of the experimental setup to measure the dependence of the magnetic force and magnetic field on the relative distance. (b) The measured data and fitted curves of the magnetic force and the magnetic field.

As expected, the plotted curves maintain the similar shape but the peak positions shift correspondingly. The inherence of the curve shapes originates from the aforementioned matched magnetic orientation. The total restoring force and the potential energy are calculated in Eq. (3) and presented in Fig. 3(c) and (d) for the following numerical simulation and parametric analysis.

$$
\left\{\begin{array}{l}
F_{\text {Tot_res }}(x)=F_{\text {Mag_res }}(x)+k x \\
E_{\text {potential }}=-\int F_{\text {Tot_res }} x d x
\end{array} .\right.
$$

Where $k$ is the linear spring constant of the cantilever beam. 

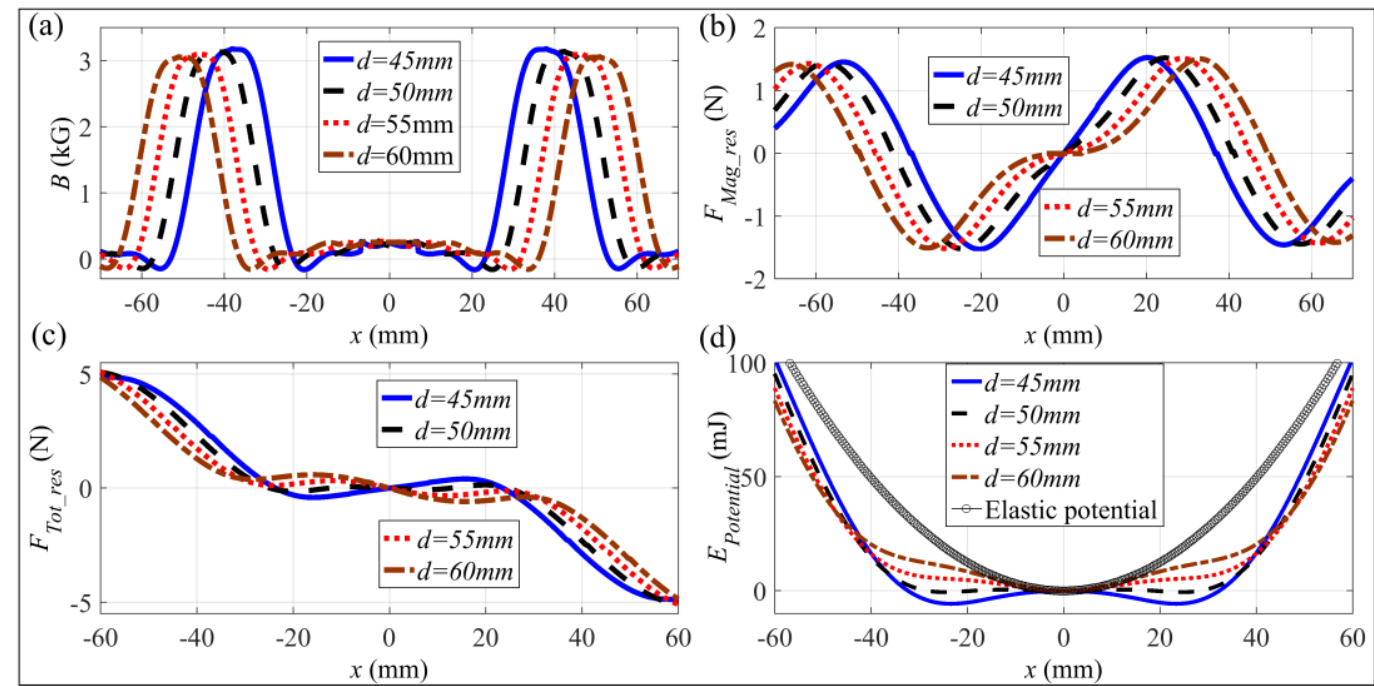

Fig. 3. Calculated (a) magnetic field, (b) magnetic restoring force, (c) total restoring force and (d) potential energy at different $d . d_{0}$ is set as $19 \mathrm{~mm}$ in the measurement.

As shown in Fig. 3(c), the nonlinear EMEH at $d=50 \mathrm{~mm}$ and $55 \mathrm{~mm}$ is expected to be capable of achieving interwell oscillations or high-energy orbits under low-frequency excitations because the corresponding restoring force curves are nearly flat with a small displacement $(-25 \mathrm{~mm}<\mathrm{x}<25 \mathrm{~mm})$, revealing that the cantilever beam is softened. In addition, according to Cao et al.'s statement the potential energy with shallower well depth produces better output performance [19], the EMEH at $d=50 \mathrm{~mm}$ will yield an enhanced output performance. The calculation results for the potential energy in Fig. 3(d) indicate that the proposed EMEH is a tristable when $d$ is relatively large. The results also show that while $d$ increases, it transforms to bistable and finally monostable.

In order to verify the calculation, the magnetic field and the total restoring force are measured at $d=50 \mathrm{~mm}$ and $d_{0}=19 \mathrm{~mm}$, as shown in Fig. 4, in comparison to the aforementioned calculated results. Although there is a slight discrepancy observed at displacements greater than $60 \mathrm{~mm}(x>60)$ this is possibly a result from a fitting error in Eq. (1). Generally, both calculated results and measured data coincide with each other, which demonstrates the accuracy of the calculation and thus laying a foundation for the following numerical simulation.
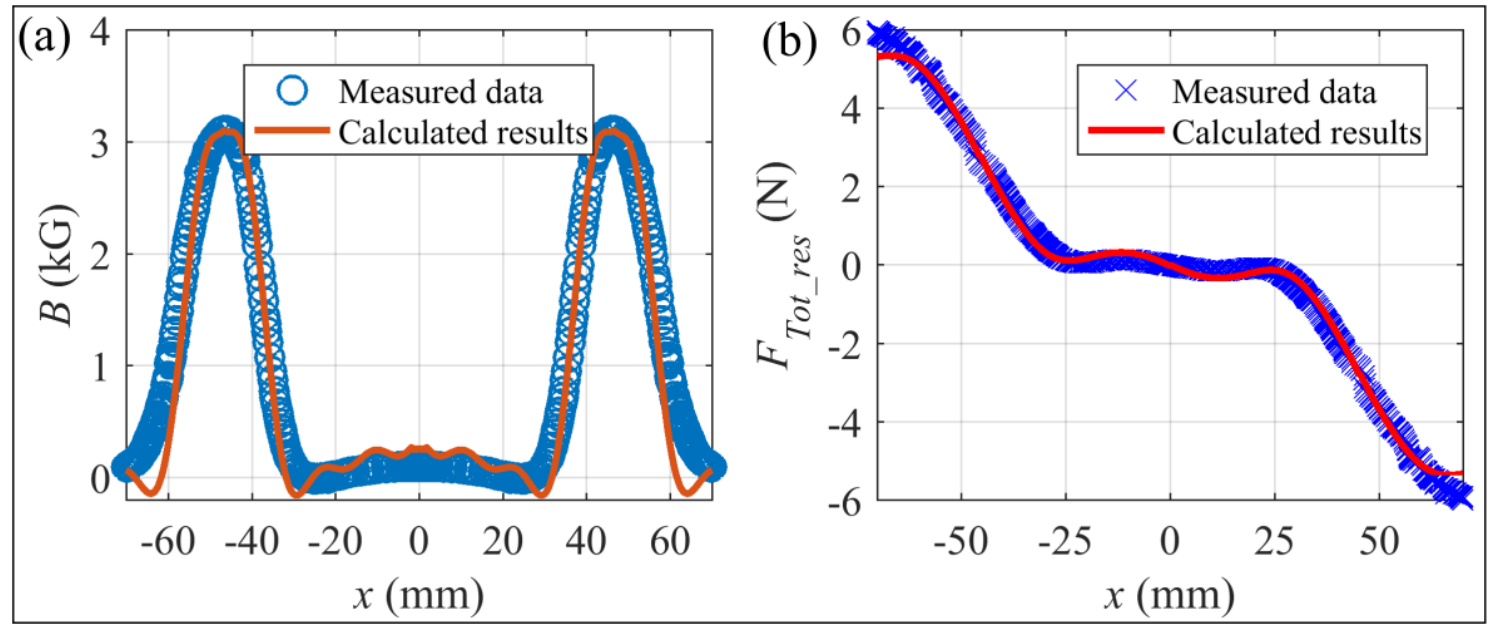

Fig. 4. Measured and calculated (a) magnetic field and (b) restoring force at $d=50 \mathrm{~mm}, d_{0}=19 \mathrm{~mm}$.

\section{Numerical simulation and experimental output of the electromagnetic voltage}


The electromechanical governing equation of the EMEH could be derived from Euler-Lagrange equation [4]:

$$
\ddot{x}+2 \zeta \omega_{0} \dot{x}=\left(F_{e x c}+F_{r e s}\right) / M
$$

Where $\zeta$ is the damping coefficient and $\omega_{0}$ is the fundamental resonant frequency. $F_{\text {exc }}$ and $F_{\text {res }}$ correspond to the excitation force and restoring force, respectively. The excitation force used in the simulation is $0.113 \mathrm{~N}$, which means the acceleration level is $0.45 \mathrm{~g}$. The restoring force is obtained from Eq. $(1,2,3)$. $M$ refers to the effective mass, calculated as $M=33 / 140 m l+M_{t}$, where $m$ is the mass per unit length of the cantilever beam, $l$ is the length of the cantilever beam and $M_{t}$ is the tip mass. The electromechanical coupling is usually dealt as equivalent damping [23]:

$$
c_{\text {Mag }}=\frac{(N B L)^{2}}{R_{\text {load }}+R_{\mathrm{int}}} .
$$

Here, $R_{\text {load }}$ and $R_{\text {int }}$ denote the external load resistance and the internal resistance of the coils, respectively. $L$ represents the total length of the coil. Though the measured maximum magnetic field is about $0.3 \mathrm{~T}$, due to the fact that the average magnetic field in the coil is very small and the magnetic field is largely dependent on the distance, the calculated damping from electromagnetic induction is much smaller than the mechanical damping and is therefore negligible. The electromagnetic voltage output is calculated through Faraday's law of induction as

$$
V_{\text {out }}=-\eta N \frac{\partial \phi}{\partial t}=-\eta N A \dot{x} \frac{\partial B}{\partial x}
$$

Here, $N$ refers to the number of turns in the coil and $A$ represents the cross section area of the coil. $\eta$ takes the average effect of magnetic field across the coil into consideration and it is set as 2e-5 from experimental identification. Eq. (6) indicates that $d$ is critical to the electromagnetic voltage output, as it determines the magnetic force and thus the nonlinear dynamics of the cantilever beam, which is shown through its influence on $\dot{x}$. Simultaneously, $d$ also determines the magnetic field on the coil and $B$ in Eq. (6) displays its effect The numerical simulation of Eq. (4) is performed using an embedded ODE solver ode45 in MatLab and then the simulated data of $x$ and $\dot{x}$ are substituted into Eq. $(1,2,6)$ to simulate the voltage output. The simulated results of upward frequency sweep are shown in Fig. 5. The downward sweep results are not shown here because they are undesirable compared to the upward sweep results due to the softened configuration. It can be seen that the output at $d=50 \mathrm{~mm}$ and $55 \mathrm{~mm}$ has a significantly larger bandwidth and higher peak value, which indicates that the inter-well oscillations were achieved. It corresponds with the aforementioned analysis based on the calculated total restoring force and potential energy that the tristable harvester with a shallow well depth has enhanced output performance[19] However, when $d=45$ or $60 \mathrm{~mm}$, the voltage output was very low due to either the vibration amplitude of the cantilever tip being too small or the magnetic field on the coil being too weak, which resulted in small magnetic flux variation. 


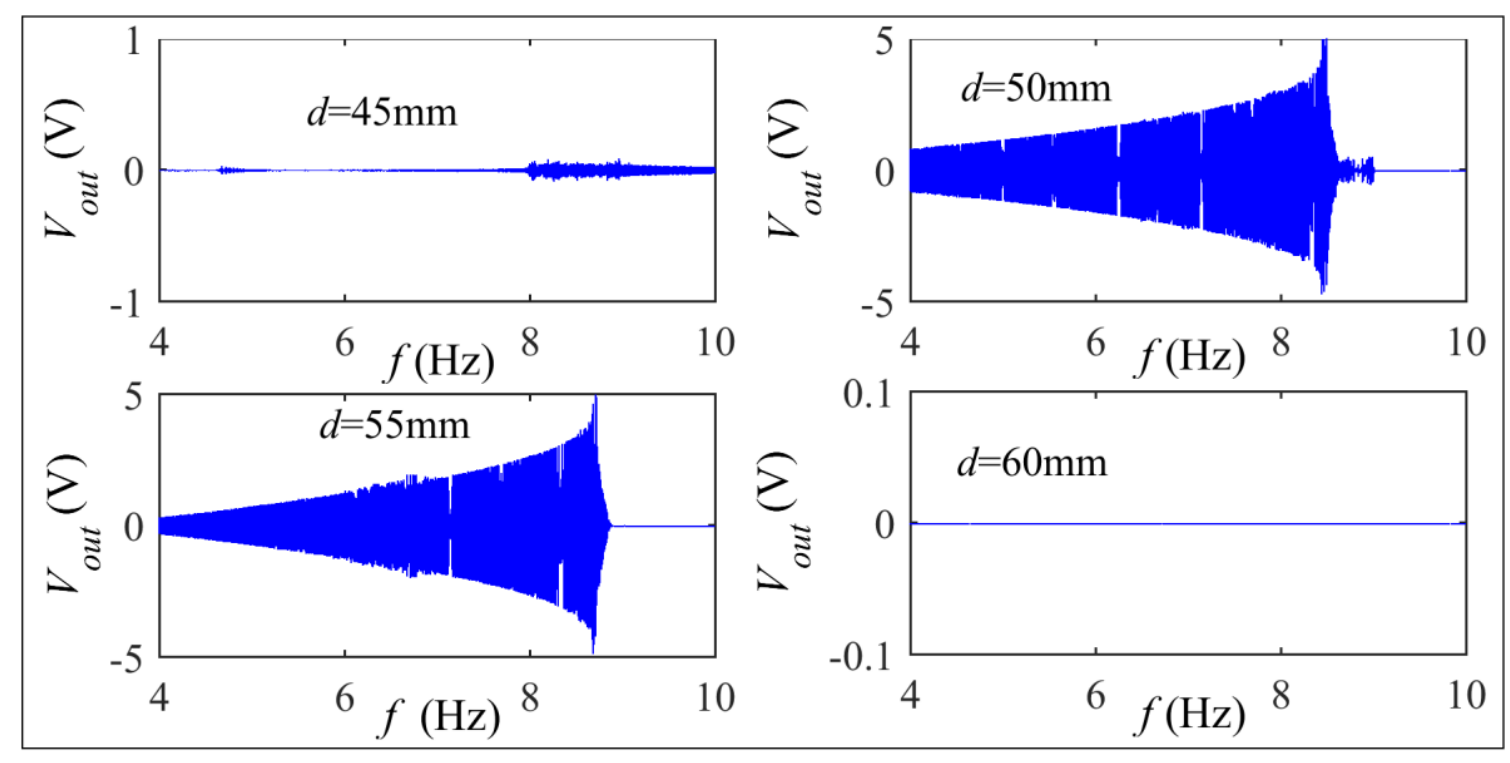

Fig. 5. Numerical simulation of electromagnetic voltage output at $d=45,50,55$ and $60 \mathrm{~mm}$.

The harvester is also investigated experimentally. The photo representation of the experimental setup is presented in Fig. 6. Labview and NI-DAQ (NI USB-6212) are used for signal generation and data acquisition. An electrodynamics shaker (APS 113) and a power amplifier (APS 125) provided the input excitation and the acceleration level was measured by an accelerometer (PCB 353B33) and was set to be $0.45 \mathrm{~g}$. The load resistance was also set as $1 \mathrm{M} \Omega$. The experimental results are presented in Fig. 7 which shows the presence of errors between the simulation results and experimental results at $d=45 \mathrm{~mm}$ and $60 \mathrm{~mm}$. One possible cause is that the magnetic flux density has a small discrepancy between the calculated data and measured data. The other cause is that the value of $d$ could have a slight influence on the tip motion trajectory of the cantilever beam and made an imperfect match to the magnetic orientations. Despite the errors at $d=45 \mathrm{~mm}$ and $60 \mathrm{~mm}$, the simulation provided a good prediction of the output at $d=50$ and $55 \mathrm{~mm}$, which are the cases of interest since it was able to achieve a high output voltage. Both the simulation and experimental results show the essential role of $d$ in the design of the EMEH.

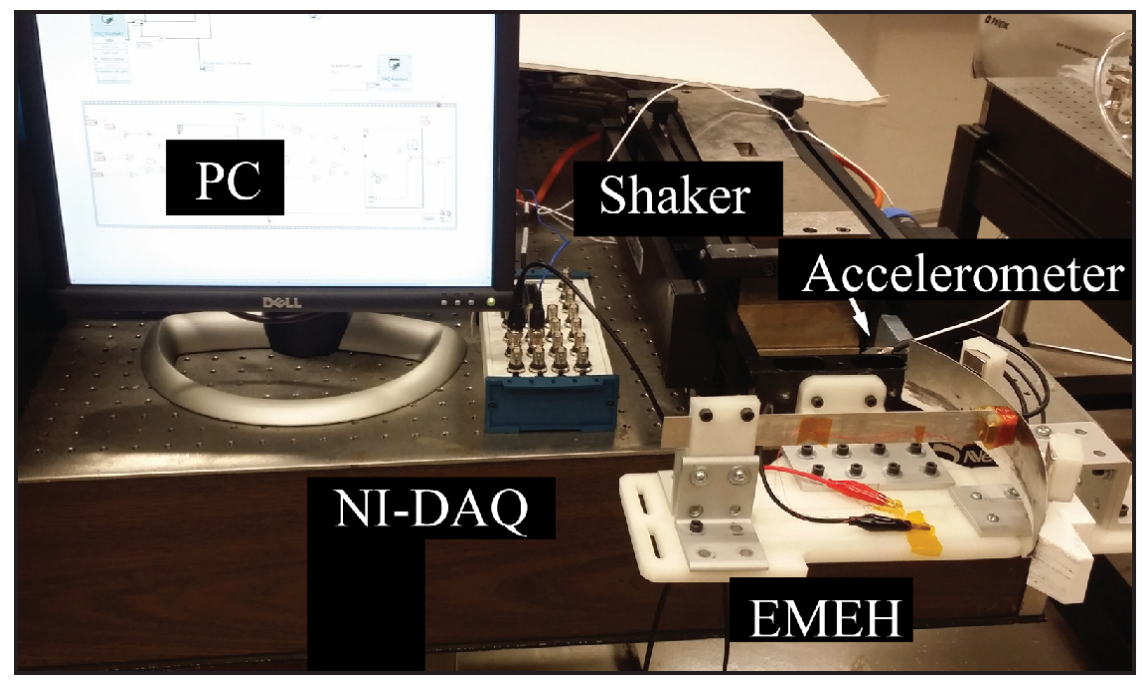

Fig. 6. A photo representation of the experimental setup. 


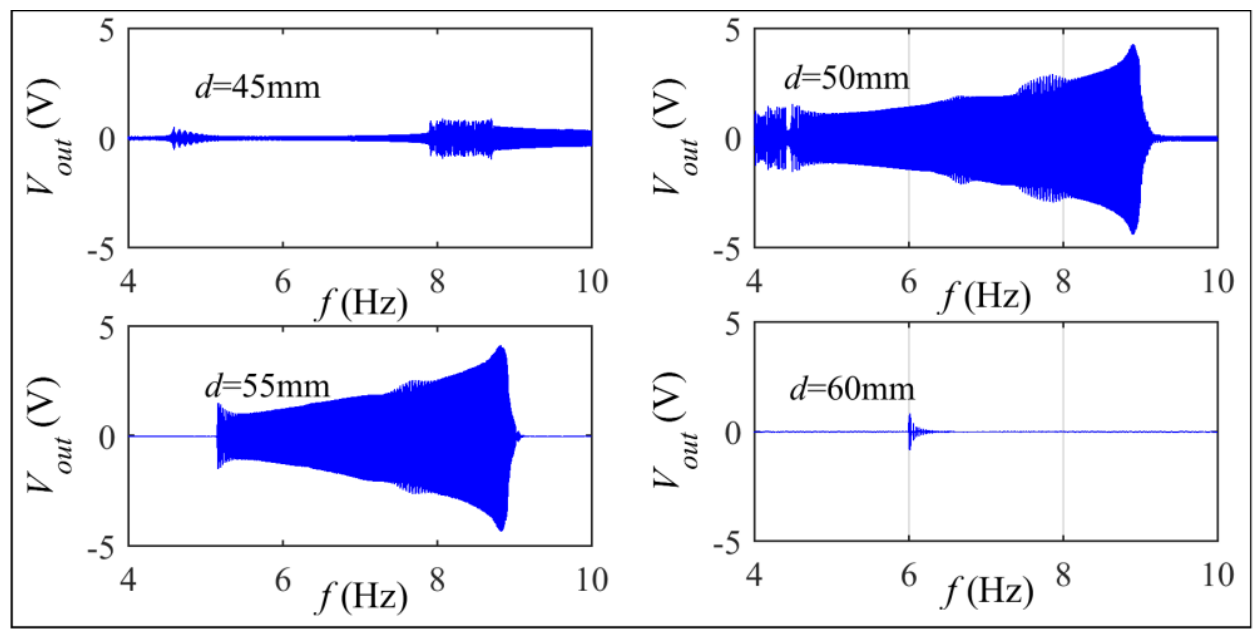

Fig. 7. Experimental measurement of electromagnetic voltage outputs at $d=45,50,55$ and $60 \mathrm{~mm}$.

The aforementioned simulation and experiments set $d_{0}$ as $19 \mathrm{~mm}$ and was able to produce a better performance at $d=50 \mathrm{~mm}$ and $55 \mathrm{~mm}$. In order to study the dependence of the performance on both $d_{0}$ and $d$, the EMEH is tested at different $d_{0}$ and $d$. To generalize the design criteria, we define the values of the parameter pair $\left(d_{0}, d\right)$ as the suitable values if the EMEH could achieve inter-well oscillations or high-energy orbits, more specifically, if the peak of the RMS voltage output is higher than $1 \mathrm{~V}$, the values of the parameter pair $\left(d_{0}, d\right)$ are regarded as suitable. For instance, the suitable values of the parameter pair $\left(d_{0}, d\right)$ could be $(19,55)$ and $(19,55)$ as concluded from Fig. 7. In addition to $d_{0}=19 \mathrm{~mm}$, experiments are also performed at $d_{0}=16.5 \mathrm{~mm}, 17.5 \mathrm{~mm}$ and $20 \mathrm{~mm}$. The measured voltage outputs (RMS) are shown in Fig. 8. As the magnetic field decreases with the distance, the peak value of voltage output decreased with the increasing of $d_{0}$. For the cases in which inter-well oscillations were achieved, the cantilever tip would have a small velocity when facing the side magnets at large value of $d$, due to the fact that the velocity of an oscillator is small at large displacements. This then resulted in the peak value of the output dropping slightly with the increasing values of $d$. Another interesting point is that $d$ has a significant influence on the jump-up point and bandwidth of the output, which is clearly shown in Fig. 8 . The jump-up point and bandwidth increase with $d$. According to Fig. 3(c) and (d), the side magnets have a small effect on the restoring force and potential energy when the cantilever tip has small displacement. In order to jump up to the high-energy orbit with the assistance of the magnetic force, the cantilever needs to have a large displacement when $d$ is large; therefore, it would require the displacement threshold to increase with increasing values of $d$.

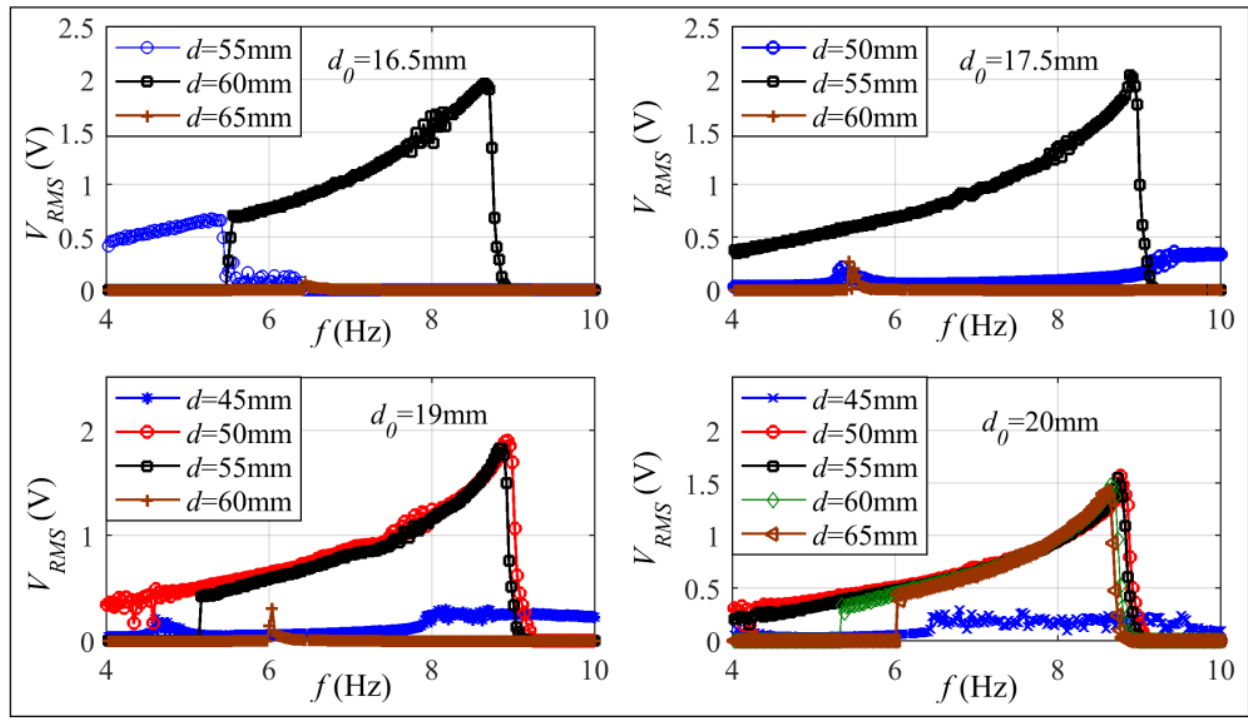

Fig. 8. Measured voltage output (RMS) at different values of the parameter pair $\left(d_{0}, d\right)$. 
Since the jump up frequency is at the left of the fundamental resonant frequency, the displacement amplitude increases with the frequency, which requires the frequency threshold to also increase with $d$ in order for the jump up to occur. This is consistent to the analysis in Ref [20] which mentions that in a tristable energy harvester, the threshold for inter-well oscillation to occur can be moved forward to the low frequency.

The suitable values of the parameter pair $\left(d_{0}, d\right)$ summarized from Fig. 8 are shown in Fig. 9. When suitable values are chosen for the design, the EMEH is capable of yielding a high voltage output. The parameter pair values located inside the shaded area and on the edges are suitable, however, it should be noted that the edges do not reflect the exact boundary because the change of $d$ in the experiments is $5 \mathrm{~mm}$ which is not small enough to provide an exact boundary. The shaded area is mainly lying in the region with large values of $d_{0}$, suggesting that the performance of the EMEH is less sensitive on the design parameters $(d, h, \alpha)$ when $d_{0}$ is large. As we know, the magnetic force is highly dependent on distance, and as $d_{0}$ determines the minimum distance between the tip magnets and side magnets, it would also determine the decreasing rate of the magnetic force. When $d_{0}$ is large, the decreasing rate is small, which means the influence of $d$ on the force is small. Correspondingly while $d_{0}$ is small, the decreasing rate is large and $d$ would have a significant influence on the force. Therefore, $d$ has a wide range of suitable values when $d_{0}$ is large. From this, two useful implications could be drawn from Fig. 9. The first one being that when we are searching for the suitable values of the parameters $(d, h, \alpha)$, it is beneficial to start from a large $d_{0}$ since the chance to find suitable values are high. The other, being that it would be better to choose a large $d_{0}$ for the design to make the harvester performance more tolerant to the manufacture imperfections.

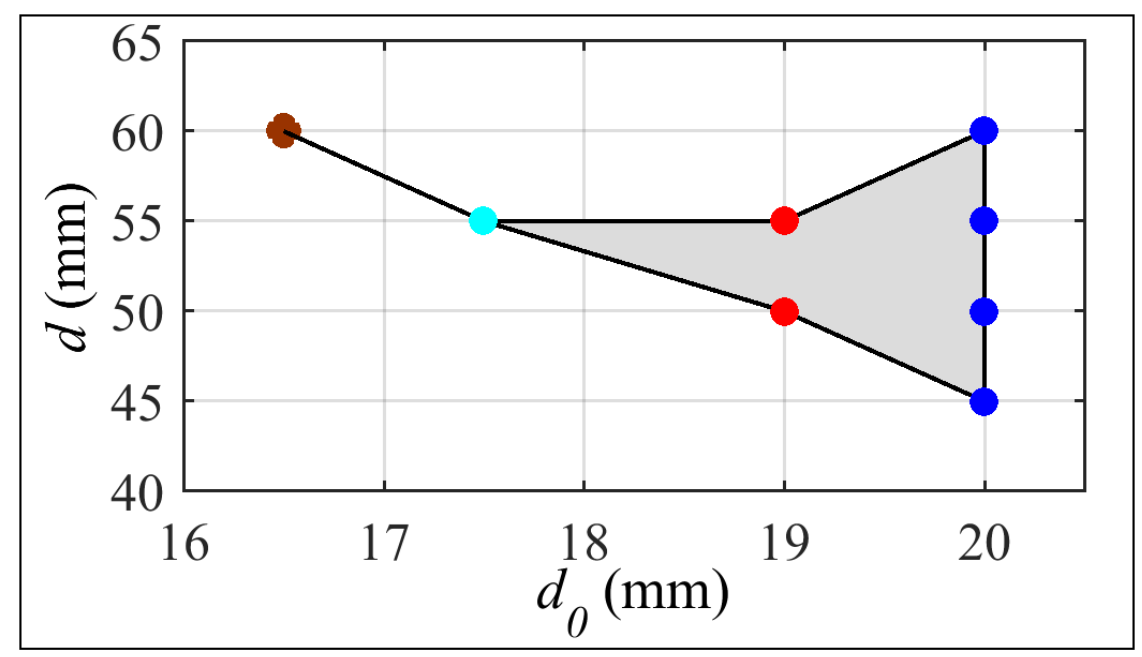

Fig. 9. The suitable values of the parameter pair $\left(d_{0}, d\right)$ obtained from the experimental results shown in Fig. 8 .

In order to demonstrate the harvesting capacity of the EMEH, it is necessary to find the optimal load resistance and optimal power output of the nonlinear EMEH. The RMS power output at different load resistances are measured at $8 \mathrm{~Hz}$ and is shown in Fig. 10 (a). Considering the fact that the harvester is operating under lowfrequency excitation and $N$ is relatively small, it is acceptable to assume that the inductive impedance of the coil is much smaller than the resistance of the copper wire so that the total impeance is independent on the frequency. As discussed earlier, the electromagnetic induction has negligible impact on the vibration, therefore the power output could be expressed as:

$$
P_{R M S}=V_{R M S}^{\text {open }} \frac{R_{\text {Load }}}{\left(R_{\text {Load }}+R_{\mathrm{int}}\right)^{2}} .
$$

Here, $V_{R M S}^{\text {open }}$ is the RMS voltage output at an open circuit. Since the frequency sweep is performed at $1 \mathrm{M} \Omega$ load resistance, which is much larger than the internal resistance, the measured voltage output could be approximated as an open circuit voltage. The fitted curve based on Eq. (7) is shown in Fig. 10 (a), which shows that the measured data and the fitted curve coincides with each other thus verifying the reasonbility of our assumption. 
Fig 10 (a) also shows that the internal resistance is found to be $22.5 \Omega$. The optimal RMS power output in the frequency domain is then calculated by

$$
P_{R M S}^{o p t}=\frac{\left(V_{R M S}^{\text {open }}\right)^{2}}{4 R_{\mathrm{int}}} .
$$
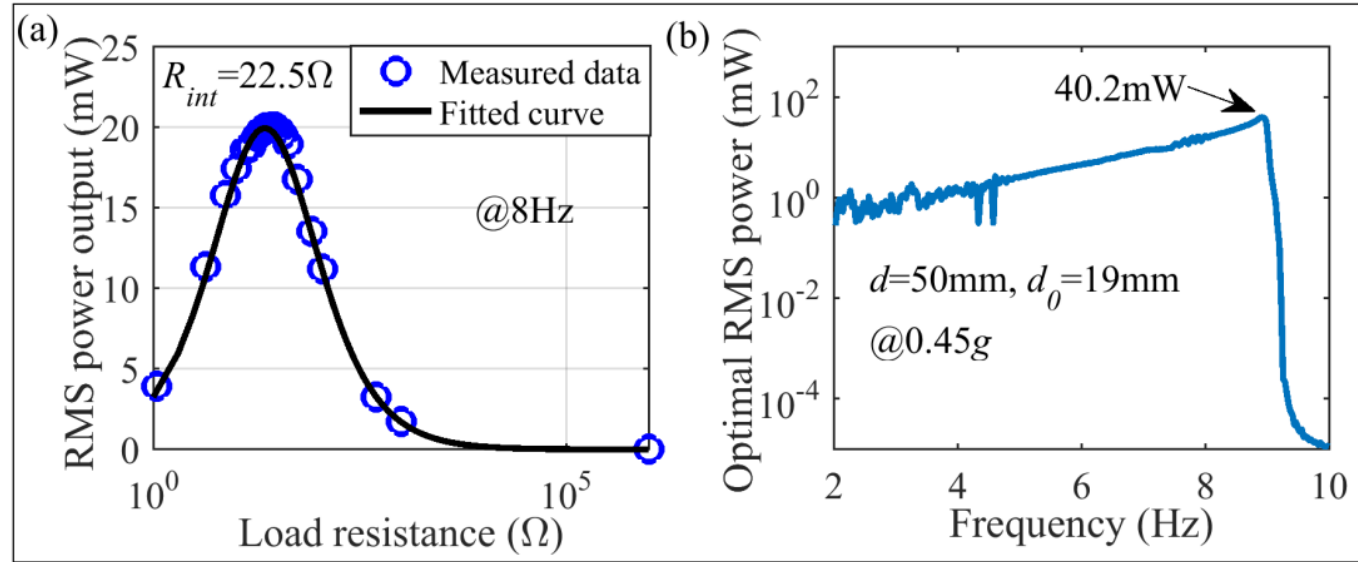

Fig. 10. (a) The dependence of power output (RMS) on the load resistance. (b) The optimal power output (RMS) in frequency domain.

The results are plotted in Fig. 10 (b), which displays the maximum power output (RMS) to be $40.2 \mathrm{~mW}$ at 8.9 $\mathrm{Hz}$ under a $0.45 \mathrm{~g}$ base excitaiton and also shows that the broadband response is achieved. According to Eq. (2) in Ref [25], the maximum available motion power is calculated as

$$
P_{\max }=\frac{2}{\pi} Y_{o} X_{l} \omega^{3} M
$$

Where $Y_{0}$ is the excitation displacement, $X_{l}$ is the maximum mass displacement and $\omega$ is the excitation angular frequency. When the excitation frequency is $8.9 \mathrm{~Hz}, Y_{0}$ is $1.41 \mathrm{~mm}$ and $X_{l}$ is $61 \mathrm{~mm}$, therefore the maximum available motion power $P_{\max }$ is $246 \mathrm{~mW}$ and the conversion efficiency from kinetic energy to electrical energy is $16.3 \%$. Since the volume and mass of the EMEH are $400.7 \mathrm{~cm}^{3}$ and $123.4 \mathrm{~g}$, respectively, the power density in terms of mass and volume is $0.81 \mathrm{~mW} /\left(\mathrm{kg} \cdot \mathrm{cm}^{3}\right)$.

\section{Conclusions}

In this paper we propose a nonlinear EMEH originating from a typical configuration of a multi-stable NEH to study the position parameters of the side magnets. The nonlinear EMEH is designed to have a matched magnetic orientation between the tip magnets and the side magnets by positioning the side magnets in the concave surface parallel to the trajectory of the cantilever tip, so that the position parameters $(d, h, \alpha)$ are inter-related by the trajectory function and could be studied interdependently. The $d$ is selected as the primary parameter $(h$ and $\alpha$ are then determined). Therefore, instead of the ternary position parameters $(d, h, \alpha)$, a binary parameters pair $\left(d_{0}\right.$, $d$ ) is used to characterize the position of the side magnets and the performance of the EMEH. Because of the well-designed structure, the magnetic force and magnetic field at the tip only depend on the relative distance in $x$-axis between the tip magnets and side magnets. Therefore, the magnetic force (field)-distance relation is measured to calculate the corresponding restoring force and magnetic field at different values of $d$. A systematic parameter study is performed by a numerical simulation based on the electromagnetic governing equation and the simulation results coinciding with the experimental results. The EMEH is also tested under different values of the parameter pair $\left(d_{0} d\right)$ and the summarized diagram of the defined suitable values of the parameter pair $\left(d_{0}\right.$, d) provided useful information about the EMEH design. The internal resistance has been found and the optimal 
power output (RMS) in the frequency domain is also obtained to demonstrate the energy harvesting capabilities of the nonlinear EMEH.

\section{Acknowledgements}

The authors would like to thank the support of U.S. DOE ARPA-E under grant DOE-AR0000531 and the support of U.S. Office of Naval Research under grant N000141410230.

\section{References}

1. Williams, C. and R.B. Yates, Analysis of a micro-electric generator for microsystems. sensors and actuators A: Physical, 1996. 52(1): p. 8-11.

2. Sodano, H.A., D.J. Inman, and G. Park, Comparison of piezoelectric energy harvesting devices for recharging batteries. Journal of Intelligent Material Systems and Structures, 2005. 16(10): p. 799-807.

3. Shu, Y. and I. Lien, Analysis of power output for piezoelectric energy harvesting systems. Smart materials and structures, 2006. 15(6): p. 1499.

4. $\quad$ Erturk, A. and D.J. Inman, Piezoelectric energy harvesting. 2011: John Wiley \& Sons.

5. Bilgen, O., Y. Wang, and D.J. Inman, Electromechanical comparison of cantilevered beams with multifunctional piezoceramic devices. Mechanical Systems and Signal Processing, 2012. 27: p. 763 777.

6. Wang, X., et al., Coupling analysis of linear vibration energy harvesting systems. Mechanical Systems and Signal Processing, 2016. 70: p. 428-444.

7. Beeby, S.P., et al., A micro electromagnetic generator for vibration energy harvesting. Journal of Micromechanics and microengineering, 2007. 17(7): p. 1257.

8. Yang, B., et al., Electromagnetic energy harvesting from vibrations of multiple frequencies. Journal of Micromechanics and Microengineering, 2009. 19(3): p. 035001.

9. Rahimi, A., et al., An electromagnetic energy harvesting system for low frequency applications with a passive interface ASIC in standard CMOS. Sensors and Actuators A: Physical, 2012. 188: p. 158-166.

10. Cottone, F., H. Vocca, and L. Gammaitoni, Nonlinear energy harvesting. Physical Review Letters, 2009. 102(8): p. 080601.

11. Gammaitoni, L., I. Neri, and H. Vocca, Nonlinear oscillators for vibration energy harvesting. Applied Physics Letters, 2009. 94(16): p. 164102.

12. Erturk, A., J. Hoffmann, and D. Inman, A piezomagnetoelastic structure for broadband vibration energy harvesting. Applied Physics Letters, 2009. 94(25): p. 254102.

13. Yang, J., Y. Xiong, and J. Xing, Nonlinear power flow analysis of the Duffing oscillator. Mechanical Systems and Signal Processing, 2014. 45(2): p. 563-578.

14. Stanton, S.C., C.C. McGehee, and B.P. Mann, Nonlinear dynamics for broadband energy harvesting: investigation of a bistable piezoelectric inertial generator. Physica D: Nonlinear Phenomena, 2010. 239(10): p. 640-653. 
15. De Paula, A.S., D.J. Inman, and M.A. Savi, Energy harvesting in a nonlinear piezomagnetoelastic beam subjected to random excitation. Mechanical Systems and Signal Processing, 2015. 54: p. 405416.

16. Stanton, S.C., C.C. McGehee, and B.P. Mann, Reversible hysteresis for broadband magnetopiezoelastic energy harvesting. Applied Physics Letters, 2009. 95(17): p. 174103.

17. Zhou, S., et al., Enhanced broadband piezoelectric energy harvesting using rotatable magnets. Applied Physics Letters, 2013. 102(17): p. 173901.

18. Zhou, S., et al., Broadband tristable energy harvester: Modeling and experiment verification. Applied Energy, 2014. 133: p. 33-39.

19. Cao, J., et al., Influence of potential well depth on nonlinear tristable energy harvesting. Applied Physics Letters, 2015. 106(17): p. 173903.

20. Haitao, L., et al., Dynamics and coherence resonance of tri-stable energy harvesting system. Smart Materials and Structures, 2015. 25(1): p. 015001.

21. Cao, J., et al., Nonlinear time-varying potential bistable energy harvesting from human motion. Applied Physics Letters, 2015. 107(14): p. 143904.

22. Wang, W., et al. Efficient energy harvesting from human motion by tristable piezoelectric cantilever. in Piezoelectricity, Acoustic Waves, and Device Applications (SPAWDA), 2015 Symposium on. 2015. IEEE.

23. Mann, B. and N. Sims, Energy harvesting from the nonlinear oscillations of magnetic levitation. Journal of Sound and Vibration, 2009. 319(1): p. 515-530.

24. VILLANI, D.D., An analytic solution for the force between two magnetic dipoles. Magnetic and electrical Separation, 1998. 9: p. 39-52.

25. Mitcheson, P.D., et al., Energy harvesting from human and machine motion for wireless electronic devices. Proceedings of the IEEE, 2008. 96(9): p. 1457-1486. 